\title{
Emerging Stock Markets' Reaction to COVID-19: Can Cryptocurrencies be a Safe Haven?
}

\author{
Ahmed JERIBI \\ Faculty of Economics and Management of Mahdia-Tunisia \\ ahmedjeribi07@yahoo.fr \\ https://orcid.org/0000-0003-3029-1585
}

\section{Dhouha CHAMSA}

Faculty of Economics and Management of Mahdia-Tunisia

chamsa.dhouha3@gmail.com

https://orcid.org/0000-0002-3296-8820

\section{Yasmine SNENE MANZLI}

Faculty of Economics and Management of Mahdia-Tunisia

yasmine.snene.manzli@gmail.com

https://orcid.org/0000-0002-4904-8342

\begin{abstract}
In this study we discuss the determinants of the BRICS and GCC stock market returns during the COVID-19 outbreak. We employ the OLS regression to discern how crypto-currencies, VIX, oil, GOLD prices, and the number of COVID-19 cases and deaths, affect the Gulf and BRICS stock markets. We find that Bitcoin and Ethereum can generate benefits from portfolio diversification and hedging strategies but not from safe haven strategies for Russia, Brazil, Abu-Dhabi, Bahrain, and Qatar financial investors during the COVID-19 outbreak. Our results reveal that Gold is neither hedge nor a safe haven but is only an effective diversifier for investors during the COVID19 outbreak. The results indicated that among all the BRICS and GCC stock indexes, the expected volatility of the US stock market has an effect only on china and Kuwait financial markets. Finally, our results show that the growth rate of confirmed COVID-19 cases has a negative impact only on South Africa and Brazil stock market.
\end{abstract}

Keywords: Emerging stock markets, crypto-currencies, COVID-19 cases and deaths, Gold, WTI, VIX, OLS regression. 


\section{1- Introduction}

Since the beginning of 2020 the world witnessed dramatic changes. The COVID-19 pandemic, the oil price battle between Russia and Saudi Arabia, and the global stock market crash have destabilized the globe in the first three months of 2020. International stock markets, futures, and crude oil prices dropped successively. Since the beginning of 2020, oil prices have dropped dramatically. In fact, consumer demand decreased following the continued spread of the COVID19 pandemic and its effects on the economy, which led to a dispute between Russia and Saudi Arabia as two of the largest oil producers at the beginning of March. On April 20, 2020, the oil price collapse devastated global stocks and launched a new battlefield for pandemic-battered economies. Future oil prices have turned negative, something that has never happened since the NYMEX oil futures began trading in 1983. The report by Goldman Sachs and the IMF noted that the world economy will suffer its worst year since the Great Depression of the 1930s.

Before the appearance of the coronavirus, cryptocurrencies became a steadily maturing niche. The last decade saw a new type of Bitcoin, first introduced by Nakamoto (2008). It is a new financial asset that is a peer-to-peer electronic cash system that allows online payments to be made directly from one party to another without going through a financial institution. Bitcoin, therefore, has no connection to any authority and has no physical representation, unlike most other financial assets. The present century is the digital century. It has created a new set of guidelines and rules. In fact, GOLD has traditionally been considered a safe haven for centuries during many crises (Baur and Lucey, 2010; Beckmann et al, 2015; Klein et al, 2018). Many authors (such as Baur and Lucey (2010), Baur and McDermott (2010), among others) consider that precious metals, in particular gold, are strong alternatives for hedging due to their low correlation with other financial assets and the safe-haven asset characteristics that they may have during times of financial instability. Baur and McDermott (2010) examine the role of GOLD in the global financial system. They tested the hypothesis that GOLD is considered as a safe haven against stocks of major emerging and developing countries. They found that GOLD can be seen as a panic buy in the immediate aftermath of an extreme negative market shock. Looking at specific crisis periods, their results suggest that GOLD was a strong safe haven for most developed markets. When discussing the co-movements between the BRICS stock markets and GOLD prices, Mensi et al. (2018) find no evidence of co-movement between these stock markets and GOLD prices suggesting that GOLD may act against extreme market movements as a hedge or safe-haven asset for the BRICS. After discovering that GOLD can serve as a safe haven against the excessive market movement, Chkili (2016) assesses the implications for GOLD and stock pairs for portfolio diversification and hedging effectiveness, indicating that adding GOLD to a stock portfolio improves its risk-adjusted return.

Meanwhile, the original and top-performing digital asset Bitcoin is playing its own game, and it is weakly correlated with the other market assets (Aslanidis et al, 2019; Charfeddine et al, 2020). This has contributed to the competition between digital assets and GOLD on the status of being a safe-haven asset. Given the current international macro-economic environment, a crash in the cryptocurrency demand is anticipated. Though, it won't be the end of the crypto. Indeed, governments are adopting fiscal measures to respond to the situation, which would have a positive impact on the crypto market. Bouri et al (2017) used a dynamic conditional correlation model to sort out whether Bitcoin can be served as a hedge safe and hedge refuge for several financial assets, which include stocks, bonds, currencies, and commodities. Their empirical results indicate that Bitcoin is an effective diversifier against movements in all the assets and they provide evidence that Bitcoin is a poor hedge and is suitable for diversification purposes only. Dyhrberg (2016) analyzes the attractiveness of Bitcoin to investors and tests its hedging properties, and concludes that this cryptocurrency has hedging capabilities and can be used in a 
portfolio to reduce the adverse effects of potential risks. Using an hourly frequency, Urquhart and Zhang (2019) looked at the safe haven and hedging properties of Bitcoin and notice that it acts, respectively, as a hedge, diversifier, and safe haven for several international currencies. Also, using a cross-quantilogram method, Shahzad et al. (2019) describe the safe haven characteristics and find some proof that Bitcoin, gold, and the commodity index studied are weak safe havens, but this behavior is time-varying.

In the last century, most of the emerging countries have experienced rapid economic growth. These economies include the BRICS countries, i.e. Brazil, Russia, India, China, and South Africa that are known for their strong impact on regional affairs. They are the five most ambitious and the largest developing economies in the world. In addition to economic growth, the BRICS stock markets deliver higher and more stable average returns (Jiang et al, 2019). The connection between domestic stock exchanges and a wave of financial reforms, such as the implementation of insider trading laws and the use of electronic trading, has also taken place in these countries. Consequently, a variety of international investors, policymakers and portfolio managers have paid attention to BRICS stock markets. Basher and Sadorsky (2016) used DCC, ADCC, and GO-GARCH to model volatilities and conditional correlations between emerging market stock prices, oil prices, VIX, GOLD prices, and bond prices, and they compare the hedge ratios of the different models. The key finding of this study is that oil is the most powerful hedge for emerging market stock prices. Using the DCC-GJR-GARCH model, Jiang et al (2019) analyzed the risk spillovers between the BRICS stock markets and the markets of precious metals. They found that the dynamic volatility ties between the precious metals and BRICS stock sectors are long-lasting and fluctuating dramatically between January 2001 and December 2017. Their findings suggested that the conditional correlation was negative; suggesting that the risks between the BRICS stock markets can be hedged by the kind of precious metals.

Over the past two decades, GCC economies have drawn growing interest. These countries hold about $48 \%$ of the world's proven oil reserves. Saudi Arabia, the UAE, and Kuwait possess more than nine-tenths of the oil deposits. They also control $40 \%$ of the world's oil exports. The economies of the GCC still depend on the hydrocarbon sector as their main export product and source of revenue. The GCC stock markets attracted huge interest from foreign investors looking to invest in these stock markets as a result of financial liberalization (Ulussever and Demirer, 2017) and high liquidity (Akoum et al . , 2012). In addition, four GCC members (Saudi Arabia, Qatar, Kuwait, and the UAE) were added to FTSE Russell's emerging market index in 2018.

The Coronavirus pandemic, the oil price war between Russia and Saudi Arabia, has destabilized multiple world financial markets and undermined global economic development. However, during this crisis period, most investors have widely asked for safe-haven assets, as stock prices fall free as they faced systematic risk. Mensi et al. (2016) found that Gold is a strong hedge and a safe haven for all Gulf markets. In addition, the VIX index may serve as a good protector against the extreme co-movement of stock markets. It could be a powerful safe haven during periods of turmoil. These results are similar to those of Basher and Sadorsky (2016). However, Maghyereh et al. (2017), Al-Yahyaee et al. (2019) argue that Gold should be used for the diversification of portfolios. Despite the growing number of published papers investigating the financial dependency structure between crypto-currencies and stock market indices, the relationship between GCC market indices and digital assets remains unexplored. In order to fill this gap in the literature, this paper explores whether these digital financial assets can offer new opportunities for diversification and hedging of GCC portfolios.

Given the oversensitivity of the GCC and BRICS markets to the global political and financial uncertainties posed by the COVID-19 pandemic and the oil price war between Russia and Saudi 
Arabia, we seek to discern how crypto-currencies, VIX, oil and GOLD prices, as well as the number of COVID-19 cases and deaths, affect the Gulf and BRICS stock markets?

The rest of the paper is organized as follows. Section 2 presents the methodology and data. Section 3 discusses the results. Section 4 concludes.

\section{2- Methodology and data}

As the virus spreads beyond China, economic activity is increasingly impacted and financial markets are falling down. In this study, we analyze the responses of the stock prices to the increase in the number of cases and deaths caused by the COVID-19 pandemic. In addition, we studied the impact of the change in cryptocurrencies prices and Gold, which are considered in many studies as safe havens, as well as the VIX index and the oil price war between Russia and Saudi Arabia on the indices of the Gulf and BRICS stock markets.

Our regression is as follows:

$$
I N D E X_{t}=\alpha_{0}+\alpha_{1} \text { CRYPTO }_{t}+\alpha_{2} V I X_{t}+\alpha_{3} W_{t}+\alpha_{4} G O L D_{t}+\alpha_{5} C C_{t}+\alpha_{6} C D_{t}+\varepsilon_{t}()
$$

With:

$I N D E X_{t}$ : The studied index's daily return;

$V I X_{t}:$ The VIX index's daily return, as a measure of the expected volatility of the US stock market;

$W T I_{t}$ : The WTI crude oil's daily return;

$G O L D_{t}:$ The GOLD daily return;

$C C_{t}$ : The growth rate of confirmed COVID-19 cases;

$C D_{t}$ : The growth rate of confirmed COVID-19 deaths.

Daily time series data of China stock prices (SSE), Brazil (BVSPT), Russia (RTSI), South-AFRICA (JSE40), India (BSE30) and the Gulf countries such as Abu-Dhabi (ADI), Saudi-Arab (TASI), Qatar (QEAS), Oman (MSI), Kuwait (FTGPST), Bahrain (BAX) are collected from the database DataStream covering the period from September 02, 2019, to April 30, 2020. From the same database, we extracted the VIX index, WTI, and GOLD prices. Data concerning the five studied cryptocurrencies (Bitcoin, Dash, Ethereum, Ripple, and Monero) was collected from the Coin Market Cap basis. The number of cases and deaths is collected from European Centre for Disease Prevention and Control (An agency of the European Union).

The empirical study involves 171 daily observations. In order to cover the effect of the 2020 global pandemic, the research period is divided into two sub-periods. The first sub-period is the period before the COVID-19 outbreak, which goes from 02 September 2019 to 30 December 2019. The second one is the period during the COVID-19 outbreak and it goes from 31 December 2019 to 30 April 2020.

Daily returns are defined by $r_{t}=\ln \left(\frac{p_{t}}{p_{(t-1)}}\right)$, with $\mathrm{P}(\mathrm{i}, \mathrm{t})$ denoting indices I closing price at time $\mathrm{t}$. In literature, this transformation of data is usually employed for series to obtain stationary data. In addition, figures 1 and 2 confirm that the return series are stationary and it shows that the entirety of the series was more volatile before the COVID-19 period than during the COVID-19 outbreak. Notably, all the series returns in figure 2 reached their negative peaks in late February and during March 2020. 

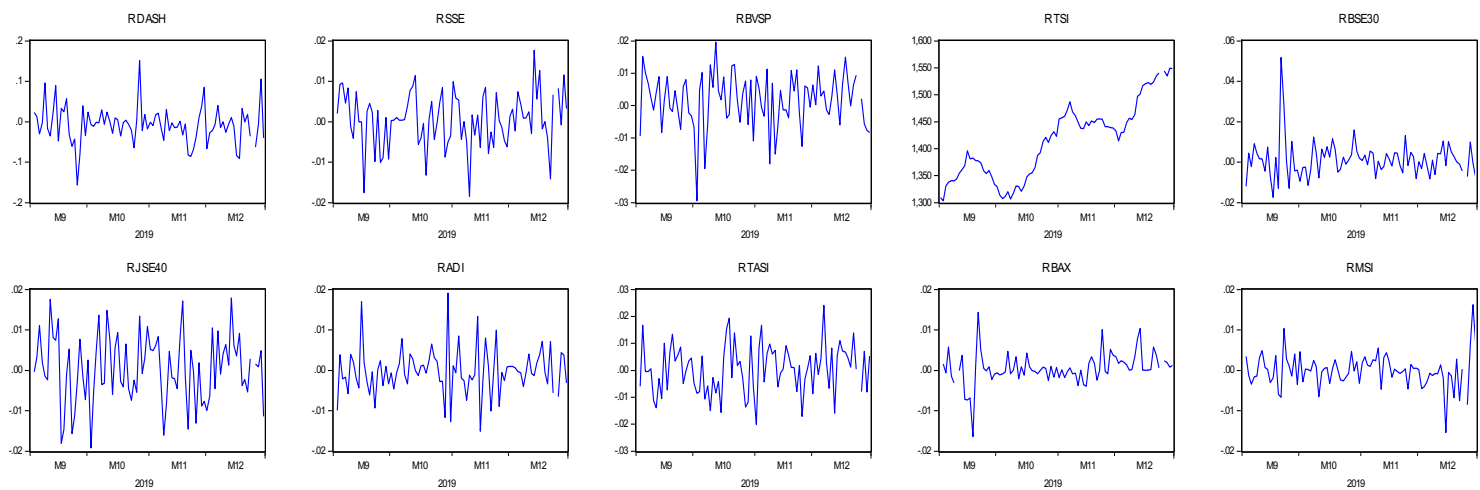

RMSI
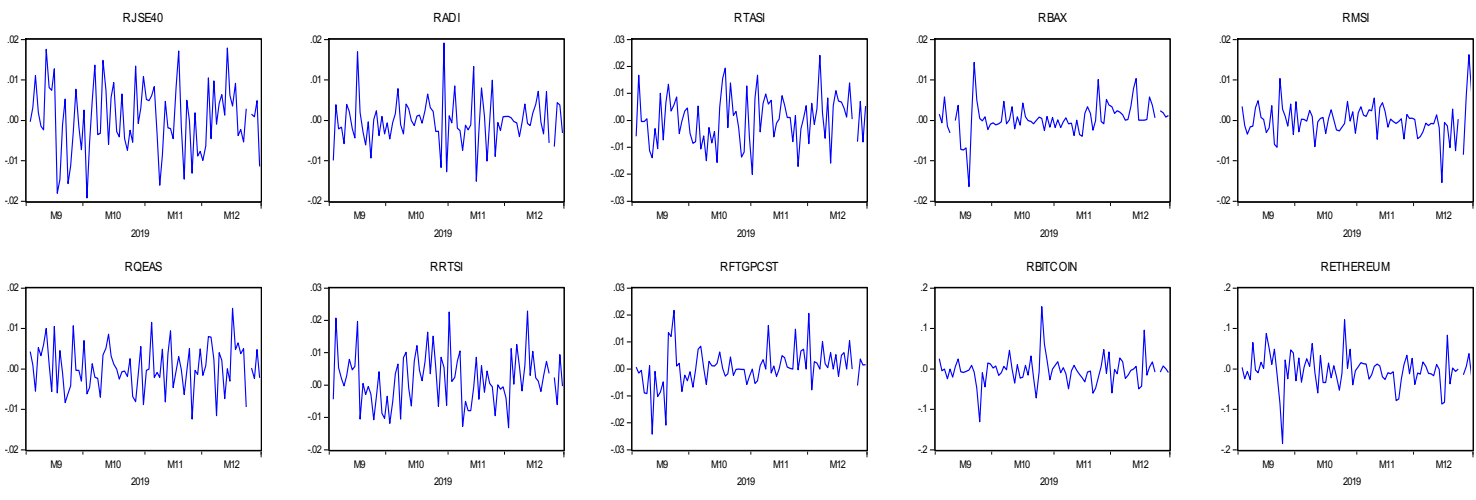

RETHEREUM
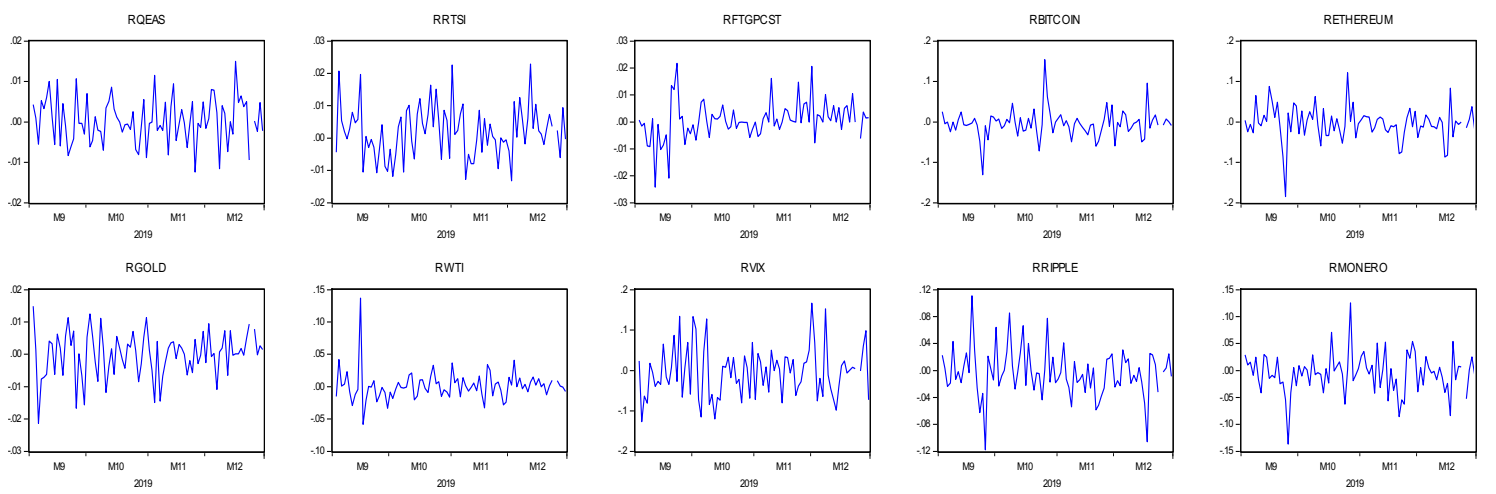

Figure 1: Evolution of series returns before COVID-19 outbreak
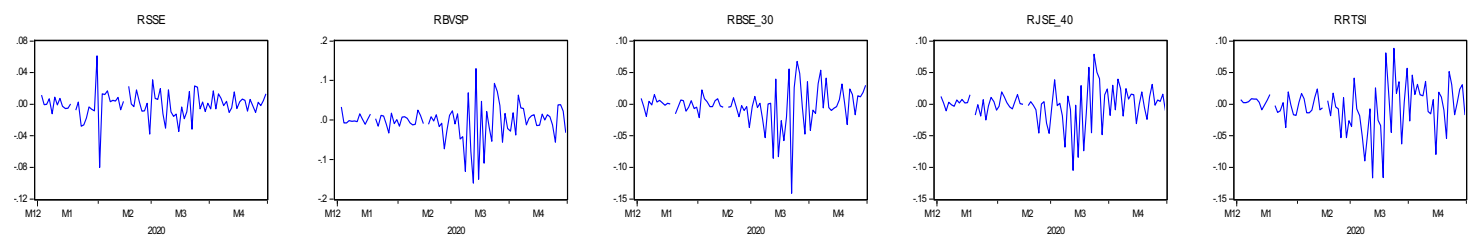

RADI

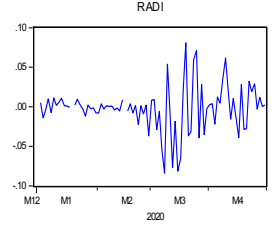

RBAX
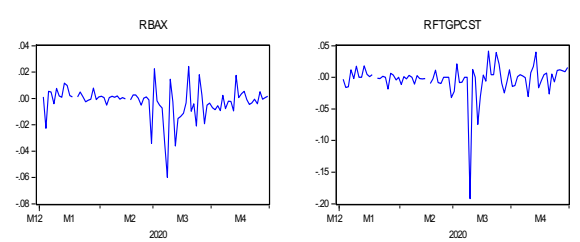

RMSI
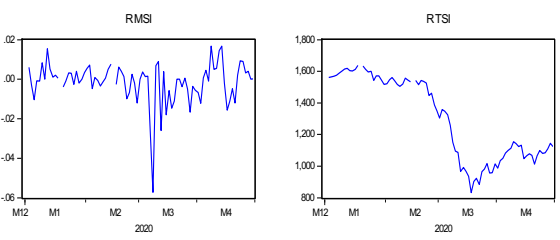

REAS
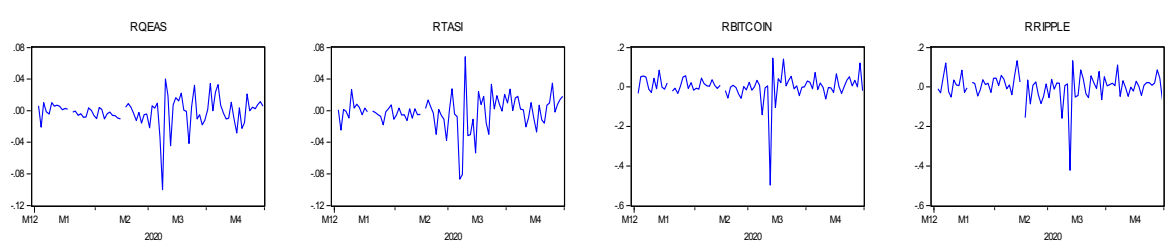

RETHEREUM
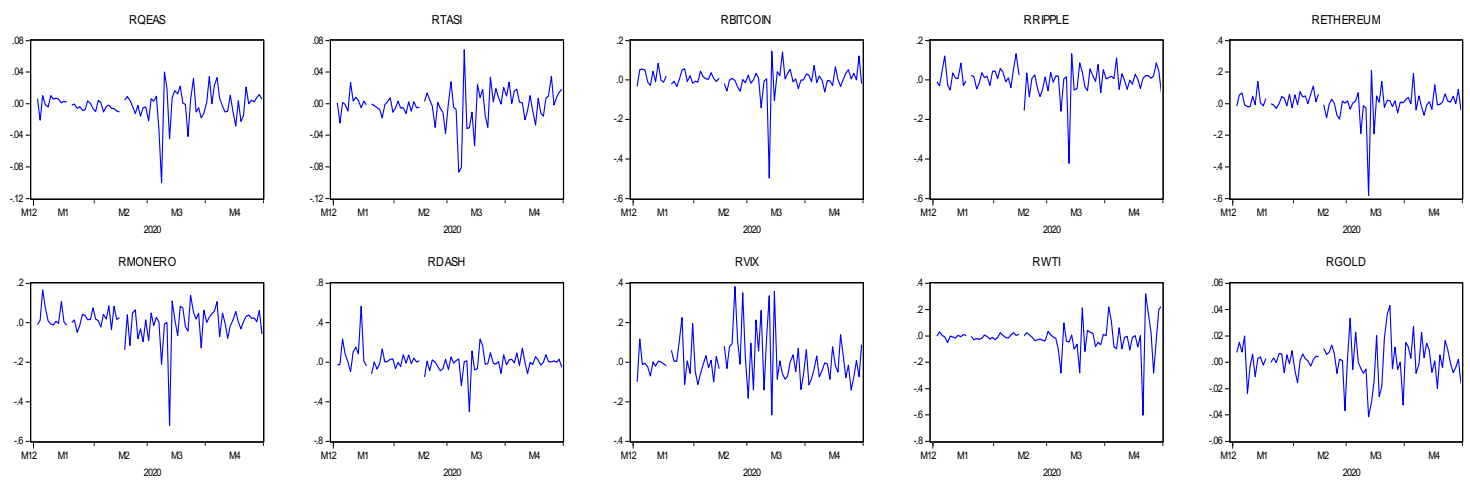

Figure 2: Evolution of series returns during COVID-19 outbreak

\section{3- Results}

The descriptive statistics of the stock market returns for the sub-periods (pre- and during COVID19 pandemic) are reported in Tables 1 and 2. 
Table 1 shows the statistic summary of the daily returns series for the BRICS and GULF stock markets, cryptocurrencies, VIX, WTI, and Gold before the COVID-19 pandemic.

As shown in Table 1, all cryptocurrencies, Gold, and VIX have a negative average value. The Russian stock market provides the highest mean return and the most volatile among the other stock markets. The standard deviations for all series except for that of Russia are higher than their mean suggesting a higher level of risk in these markets. Russia, India, Abu-Dhabi, Qatar, and Oman stock indexes, all cryptocurrencies (except for Ethereum and Monero), VIX, and Gold exhibit positive skewness, while the others are left-skewed showing a non-symmetric distribution.

As we can perceive, the kurtosis of the realized measures (excluding Russia, Arab-Saudi, and Qatar stock indices) is above three, indicating high peaks and fat tails (leptokurtic).

Table 2 provides the statistical proprieties of the considered return series during the covid-19 pandemic. Results indicate that all cryptocurrencies, VIX, and Gold have a positive average value compared to those during the pre-coronavirus. In addition, results show that all stock market indices returns have negative mean except for RTSI and JSE 40 while the other series have a positive mean. It can be seen that the highest mean of growth rates is for Brazil's cumulative number of COVID-19 cases, followed by Russia's cumulative number of COVID-19 cases.

From January to April 2020, global oil consumption decreased by 30\%, due to the COVID-19 pandemic and air carriers, which consume $8 \%$ of the global demand. In terms of standard deviation, all variables registered a higher rate during the COVID-19 pandemic than during the period before and Russia registered the highest rate among them. We also notice that all stock market indexes show a non-symmetric distribution. For all the growth rates, Dash and VIX exhibit positive skewness, while the others are left-skewed showing a non-symmetric distribution. The kurtosis coefficient values are above three for all the return series (except for Russia and South Africa), indicating high peaks and fat tails (leptokurtic) which suggest that these series are not normally distributed, which is proved by the Jarque-Bera statistics.

Table 1. Summary statistics for returns before COVID-19 outbreak

\begin{tabular}{|c|c|c|c|c|c|c|c|c|}
\hline & & Mean & Max & Min & $\begin{array}{l}\text { Std. } \\
\text { Dev. }\end{array}$ & Skewness & Kurtosis & J-B \\
\hline \multirow{8}{*}{ 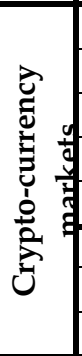 } & BITCOIN & -0.0042 & 0.1539 & -0.1306 & 0.0342 & 0.7622 & 9.4908 & $157.44^{* * *}$ \\
\hline & DASH & -0.0074 & 0.1511 & -0.1564 & 0.0456 & 0.2901 & 5.1597 & $17.7132^{* * *}$ \\
\hline & ETHEREUM & -0.0037 & 0.1212 & -0.1848 & 0.0416 & -0.6429 & 6.9904 & $62.251^{* * *}$ \\
\hline & MONERO & -0.0052 & 0.1253 & -0.1363 & 0.0367 & -0.11584 & 5.4003 & $20.595^{* * * *}$ \\
\hline & RIPPLE & -0.0032 & 0.1108 & -0.1175 & 0.0354 & 0.0842 & 5.1063 & $15.814^{* * *}$ \\
\hline & GOLD & $-7.00 \mathrm{E}-05$ & 0.0148 & -0.0213 & 0.0069 & -0.5860 & 3.5016 & $5.756^{* *}$ \\
\hline & \begin{tabular}{|l|} 
VIX \\
\end{tabular} & -0.0039 & 0.1667 & -0.1261 & 0.0629 & 0.4932 & 3.0414 & $3.453^{*}$ \\
\hline & WTI & 0.0013 & 0.1369 & -0.0582 & 0.0224 & 2.4825 & 17.4739 & $829.268^{* * *}$ \\
\hline \multirow{11}{*}{ 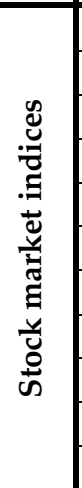 } & SSE & 0.0005 & 0.0176 & -0.0184 & 0.0067 & -0.3665 & 3.3323 & $2.2676^{*}$ \\
\hline & BVSP & 0.0015 & 0.0195 & -0.0294 & 0.0084 & -0.8090 & 4.2754 & $14.857^{* * *}$ \\
\hline & RTSI & 1415.617 & 1549.400 & 1303.490 & 69.1900 & 0.1657 & 2.0375 & $3.626^{*}$ \\
\hline & JSE 40 & -0.0010 & 0.0790 & -0.1045 & 0.0283 & -0.8321 & 5.7017 & $35.242^{*}$ \\
\hline & BSE 30 & 0.0012 & 0.0518 & -0.0174 & 0.0090 & 2.2052 & 13.6657 & $466.238^{* * *}$ \\
\hline & ADI & -0.0002 & 0.0191 & -0.0150 & 0.0056 & 0.5065 & 4.9306 & $16.638^{* * *}$ \\
\hline & TASI & 0.0007 & 0.0241 & -0.0200 & 0.0090 & -0.0064 & 2.7258 & $0.263^{*}$ \\
\hline & QEAS & 0.0002 & 0.0149 & -0.0124 & 0.0055 & 0.09050 & 2.7823 & $0.280^{*}$ \\
\hline & MSI & -0.0001 & 0.0162 & -0.0153 & 0.0041 & 0.1418 & 6.7444 & $49.355^{* * *}$ \\
\hline & FTGPCST & 0.0006 & 0.0217 & -0.0242 & 0.0071 & -0.0549 & 5.6975 & $25.511^{* * *}$ \\
\hline & BAX & 0.0005 & 0.0143 & -0.0163 & 0.0038 & -0.2211 & 8.2395 & $96.769^{* * *}$ \\
\hline
\end{tabular}


Table 2. Summary statistics for returns during COVID-19 outbreak

\begin{tabular}{|c|c|c|c|c|c|c|c|c|}
\hline & & Mean & Max & Min & $\begin{array}{l}\text { Std. } \\
\text { Dev. }\end{array}$ & Skewness & Kurtosis & J-B \\
\hline \multirow{8}{*}{$\sum_{0}^{\frac{1}{2}}$} & BITCOIN & 0.0022 & 0.1459 & -0.4972 & 0.0700 & -4.2309 & 32.4184 & $3279.675^{* * *}$ \\
\hline & DASH & 0.0076 & 0.5648 & -0.5002 & 0.1123 & 0.4421 & 13.4677 & $386.248^{* * *}$ \\
\hline & ETHEREUM & 0.0055 & 0.21063 & -0.5798 & 0.0893 & -3.2876 & 23.8354 & $1670.728^{* * *}$ \\
\hline & MONERO & 0.0037 & 0.1657 & -0.5195 & 0.0837 & -3.0929 & 19.8684 & $1129.828^{* * *}$ \\
\hline & RIPPLE & 0.0010 & 0.1334 & -0.4225 & 0.0704 & -2.6299 & 17.2855 & $811.098^{* * *}$ \\
\hline & GOLD & 0.00124 & 0.042968 & -0.0415 & 0.0146 & -0.2195 & 4.3048 & $6.633^{* *}$ \\
\hline & VIX & 0.0108 & 0.3821 & -0.2662 & 0.1191 & 1.1246 & 4.7998 & $29.044^{* * *}$ \\
\hline & WTI & -0.0140 & 0.3196 & -0.6016 & 0.1125 & -1.4378 & 12.1802 & $323.909^{* * *}$ \\
\hline \multirow{11}{*}{ 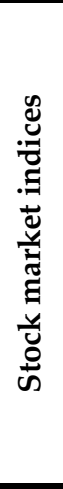 } & SSE & -0.0007 & 0.0612 & -0.0799 & 0.0172 & -0.7885 & 8.6988 & $122.375^{* * *}$ \\
\hline & BVSP & -0.0042 & 0.1302 & -0.1599 & 0.0443 & -0.8743 & 6.3742 & $50.552^{* * *}$ \\
\hline & RTSI & 1299.011 & 1637.750 & 832.2600 & 258.5898 & -0.1154 & 1.3621 & $9.575^{* * *}$ \\
\hline & JSE 40 & 0.0004 & 0.0179 & -0.0192 & 0.0084 & -0.1658 & 2.6552 & $0.801^{* * *}$ \\
\hline & BSE 30 & -0.0024 & 0.0674 & -0.1410 & 0.0299 & -1.3730 & 8.3120 & $125.157^{* * *}$ \\
\hline & ADI & -0.0021 & 0.0807 & -0.0840 & 0.0287 & -0.1681 & 4.9016 & $13.052^{* * *}$ \\
\hline & TASI & -0.0019 & 0.0683 & -0.0868 & 0.0215 & -0.9850 & 7.5409 & $85.754^{* * *}$ \\
\hline & QEAS & -0.0015 & 0.0399 & -0.0999 & 0.0181 & -1.8494 & 12.3331 & $352.759^{* * *}$ \\
\hline & MSI & -0.0014 & 0.0168 & -0.0573 & 0.0102 & -2.2368 & 12.6117 & $393.401^{* * *}$ \\
\hline & FTGPCST & -0.0030 & 0.0413 & -0.1918 & 0.0261 & -4.6453 & 34.0847 & $3684.034^{* * *}$ \\
\hline & BAX & -0.0024 & 0.0242 & -0.0600 & 0.0119 & -1.7857 & 9.5646 & $195.475^{* * *}$ \\
\hline \multirow{22}{*}{ 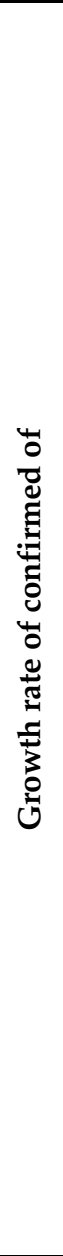 } & CC china & 0.0957 & 1.8127 & 0.0000 & 0.2558 & 4.6728 & 28.5104 & $2583.439^{* * *}$ \\
\hline & CD china & 0.1004 & 1.1363 & 0.0000 & 0.2306 & 3.2143 & 13.4011 & $523.291^{* * *}$ \\
\hline & CC Brazil & 0.1341 & 1.1394 & 0.0000 & 0.2301 & 2.5212 & 9.7439 & $248.180^{* * *}$ \\
\hline & CD Brazil & 0.1024 & 1.4271 & 0.0000 & 0.2375 & 4.1698 & 22.550 & $1581.188^{* * *}$ \\
\hline & CC Russia & 0.1287 & 0.9162 & 0.0000 & 0.2094 & 2.1721 & 7.6225 & $140.841^{* * *}$ \\
\hline & CD Russia & 0.0736 & 1.3862 & 0.0000 & 0.1884 & 4.6429 & 29.9881 & $2851.049^{* * *}$ \\
\hline & $\begin{array}{l}\text { CC South- } \\
\text { africa }\end{array}$ & 0.1022 & 1.0986 & 0.0000 & 0.2192 & 3.0847 & 12.8588 & $473.410^{* * *}$ \\
\hline & $\begin{array}{l}\text { CD South- } \\
\text { africa }\end{array}$ & 0.0469 & 0.7884 & 0.0000 & 0.1277 & 3.6395 & 17.7108 & $942.873^{* * *}$ \\
\hline & CC India & 0.1238 & 1.5404 & 0.0000 & 0.2280 & 3.6402 & 20.1991 & $1220.857^{* * *}$ \\
\hline & CD India & 0.0830 & 0.6931 & 0.0000 & 0.1582 & 2.2832 & 7.6501 & $148.668^{* * *}$ \\
\hline & CC Oman & 0.0837 & 0.7621 & 0.0000 & 0.1496 & 2.5349 & 10.0468 & $263.764^{* * *}$ \\
\hline & CD Oman & 0.0274 & 0.6931 & 0.0000 & 0.1108 & 4.6079 & 24.3559 & $1893.528^{* * *}$ \\
\hline & $\begin{array}{l}\text { CC Arab- } \\
\text { Saudi }\end{array}$ & 0.1187 & 1.6094 & 0.0000 & 0.2417 & 3.7333 & 20.1558 & $1225.260^{* * *}$ \\
\hline & $\begin{array}{l}\text { CD Arab- } \\
\text { Saudi }\end{array}$ & 0.0601 & 0.9808 & 0.0000 & 0.1583 & 3.7667 & 18.6083 & $1051.311^{* * *}$ \\
\hline & CC Bahrain & 0.0247 & 0.6931 & 0.0000 & 0.0982 & 4.9366 & 29.8657 & $2867.378^{* * *}$ \\
\hline & CD Bahrain & 0.0949 & 2.4423 & 0.0000 & 0.2857 & 6.8725 & 55.8387 & $10433.02^{* * *}$ \\
\hline & CC Kuwait & 0.0848 & 0.8602 & 0.0000 & 0.1613 & 3.0429 & 12.8044 & $466.082^{* * *}$ \\
\hline & CD Kuwait & 0.0378 & 0.8472 & 0.0000 & 0.1353 & 4.4353 & 23.4881 & $1744.592^{* * *}$ \\
\hline & CC Qatar & 0.0992 & 2.3902 & 0.0000 & 0.2949 & 6.1138 & 45.5650 & $6864.536^{* * *}$ \\
\hline & CD Qatar & 0.0274 & 0.6931 & 0.0000 & 0.1038 & 4.5819 & 25.3934 & $2049.048^{* * *}$ \\
\hline & $\begin{array}{l}\text { CC Abu- } \\
\text { Dhabi }\end{array}$ & 0.1117 & 1.3862 & 0.0000 & 0.2014 & 3.7466 & 21.6142 & $1409.242^{* * *}$ \\
\hline & $\begin{array}{l}\text { CD Abu- } \\
\text { Dhabi }\end{array}$ & 0.0463 & 0.9162 & 0.0000 & 0.1258 & 4.6645 & 29.6447 & $2789.420^{* * *}$ \\
\hline
\end{tabular}


Note: CC indicate the growth rate of confirmed COVID-19 cases while CD indicate the growth rate of confirmed COVID-19 deaths. Where St.dev represents the standard deviation of returns and JB Stat indicates the Jarque-Bera statistics.

In this study, we empirically show the impact of cryptocurrencies, VIX, oil, and Gold prices as well as the growth rates of COVID-19 cases and deaths on BRICS and Gulf countries before and during the COVID-19 outbreak. The results of the OLS regression are presented in the following tables:

Table 3. Estimated OLS regression results for BRICS countries before COVID-19 outbreak

\begin{tabular}{|c|c|c|c|c|c|c|c|c|c|c|}
\hline & C & Bitcoin & $\begin{array}{l}\text { Ethereu } \\
\text { m }\end{array}$ & Dash & Monero & Ripple & VIX & WTI & GOLD & $\mathbf{R}^{2}$ \\
\hline \multirow{5}{*}{ 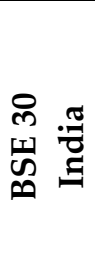 } & 0.0012 & -0.0154 & - & - & - & - & 0.0049 & 0.0275 & 0.1288 & 0.0220 \\
\hline & 0.0011 & - & -0.0373 & - & - & - & 0.0059 & 0.0336 & 0.1105 & 0.0482 \\
\hline & 0.0011 & - & - & -0.0166 & - & - & 0.0071 & 0.0256 & 0.1245 & 0.0255 \\
\hline & 0.0012 & - & - & - & -0.0001 & - & 0.0060 & 0.0299 & 0.1260 & 0.0186 \\
\hline & 0.0012 & - & - & - & - & -0.0059 & 0.0066 & 0.0296 & 0.1271 & 0.0191 \\
\hline \multirow{5}{*}{ 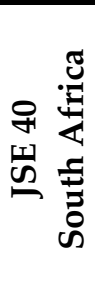 } & 0.0004 & $0.0459^{* * *}$ & - & - & - & - & -0.0119 & $0.0774^{* * *}$ & -0.0776 & 0.0941 \\
\hline & 0.0003 & - & 0.0137 & - & - & - & -0.0152 & $0.0689^{* * *}$ & -0.0637 & 0.0642 \\
\hline & 0.0004 & - & - & 0.0293 & - & - & -0.0172 & $0.0779^{* * *}$ & -0.0667 & 0.0845 \\
\hline & 0.0003 & - & - & - & 0.0132 & - & -0.0162 & $0.0705^{* * *}$ & -0.0735 & 0.0629 \\
\hline & 0.0003 & - & - & - & - & 0.0203 & -0.0172 & $0.0712^{* * *}$ & -0.0733 & 0.0668 \\
\hline \multirow{5}{*}{ 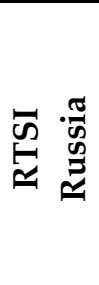 } & 0.0017 & 0.0185 & - & - & - & - & $-0.0351^{*}$ & $0.1642^{*}$ & -0.0537 & 0.3271 \\
\hline & 0.0016 & - & 0.0018 & - & - & - & $-0.0364^{*}$ & $0.1612^{*}$ & -0.0496 & 0.3210 \\
\hline & 0.0017 & - & - & 0.0167 & - & - & $-0.0375^{*}$ & $0.1657^{*}$ & -0.0489 & 0.3299 \\
\hline & 0.0016 & - & - & - & 0.0016 & - & $-0.0366^{*}$ & $0.1614^{*}$ & -0.0509 & 0.3209 \\
\hline & 0.0016 & - & - & - & - & 0.0114 & $-0.0375^{*}$ & $0.1619^{*}$ & -0.0526 & 0.3234 \\
\hline \multirow{5}{*}{ 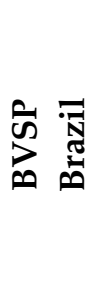 } & 0.0013 & 0.0160 & -- & - & - & - & $-0.0418^{* *}$ & $0.0727^{* * *}$ & -0.1501 & 0.1910 \\
\hline & 0.0012 & - & -0.0137 & - & - & - & $-0.0430^{*}$ & $0.0715^{* * *}$ & -0.1529 & 0.1915 \\
\hline & 0.0012 & - & - & 0.0022 & - & - & $-0.0431^{*}$ & $0.0708^{* * *}$ & -0.1470 & 0.1870 \\
\hline & 0.0013 & - & - & - & 0.0239 & - & $-0.0445^{*}$ & $0.0706^{* * *}$ & -0.1546 & 0.1976 \\
\hline & 0.0012 & - & - & - & - & -0.0040 & $-0.0426^{*}$ & $0.0700^{* * *}$ & -0.1464 & 0.1871 \\
\hline \multirow{5}{*}{ ஸี } & 0.0004 & 0.0225 & - & - & - & - & -0.0069 & $0.0656^{* *}$ & 0.0467 & 0.0646 \\
\hline & 0.0003 & - & -0.0011 & - & - & - & -0.0086 & $0.0622^{* * *}$ & 0.0502 & 0.0519 \\
\hline & 0.0005 & - & - & $0.0266^{* * *}$ & - & - & -0.0103 & $0.0690^{* *}$ & 0.0531 & 0.0835 \\
\hline & 0.0005 & - & - & - & 0.0309 & - & -0.0106 & $0.0626^{* * *}$ & 0.0411 & 0.0797 \\
\hline & 0.0004 & - & - & - & -- & 0.0288 & -0.0112 & $0.0634^{* * *}$ & 0.0451 & 0.0740 \\
\hline
\end{tabular}

${ }^{*}{ }^{* *}$ and ${ }^{* * *}$ means significant at $1 \%, 5 \%$ and $10 \%$ level of significance respectively. 
Journal of Management and Economic Studies, vol.2, issue.3, pp.152-165

Table 4. Estimated OLS regression results for Gulf countries before COVID-19 outbreak

\begin{tabular}{|c|c|c|c|c|c|c|c|c|c|c|}
\hline & C & Bitcoin & Ethereum & Dash & Monero & Ripple & VIX & WTI & GOLD & $\mathbf{R}^{2}$ \\
\hline \multirow{5}{*}{ 肴 } & 0.0005 & 0.0009 & - & - & - & - & -0.0003 & -0.0092 & 0.0741 & 0.0202 \\
\hline & 0.0005 & - & -0.0112 & - & - & - & -0.0004 & -0.0082 & 0.0696 & 0.0348 \\
\hline & 0.0004 & - & - & $-0.0157^{* * *}$ & - & - & 0.0006 & -0.0135 & 0.0725 & 0.0540 \\
\hline & 0.0005 & - & - & - & -0.0101 & & 0.0002 & -0.0096 & 0.0772 & 0.0294 \\
\hline & 0.0004 & - & - & - & - & $-0.0327^{*}$ & 0.0026 & -0.0109 & 0.0801 & 0.1078 \\
\hline \multirow{5}{*}{ 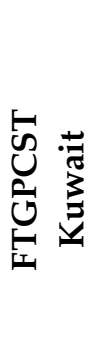 } & 0.0006 & -0.0159 & - & - & - & - & 0.0211 & 0.0130 & -0.0457 & 0.0428 \\
\hline & 0.0006 & - & -0.0288 & - & - & - & $0.0222^{* * *}$ & 0.0183 & -0.0605 & 0.0656 \\
\hline & 0.0006 & - & - & -0.0160 & - & - & $0.0233^{* * *}$ & 0.0113 & -0.0500 & 0.0474 \\
\hline & 0.0007 & - & - & - & -0.0036 & - & $0.0225^{* * *}$ & 0.0154 & -0.0474 & 0.0373 \\
\hline & 0.0005 & - & - & - & - & $-0.0543^{* *}$ & $0.0272^{* *}$ & 0.0130 & -0.0380 & 0.1089 \\
\hline \multirow{5}{*}{ 预 } & $\begin{array}{c}2.92 \mathrm{E}- \\
05\end{array}$ & 0.0039 & - & - & - & - & 0.0111 & -0.0288 & -0.0789 & 0.0621 \\
\hline & $\begin{array}{c}2.46 \mathrm{E}- \\
05\end{array}$ & - & 0.0031 & - & - & - & 0.0108 & -0.0297 & -0.0769 & 0.0620 \\
\hline & 9.99E- & - & - & 0.0128 & - & - & 0.0100 & -0.0261 & -0.0770 & 0.0807 \\
\hline & 9.83E-05 & - & - & - & 0.0174 & - & 0.0097 & -0.0291 & -0.0836 & 0.0847 \\
\hline & $\begin{array}{c}4.99 \mathrm{E}- \\
05\end{array}$ & - & - & - & - & 0.0133 & 0.0096 & -0.0288 & -0.0807 & 0.0738 \\
\hline \multirow{5}{*}{$\begin{array}{ll}\sum_{\pi}^{n} \\
\text { a }\end{array}$} & 0.0002 & -0.0044 & - & - & - & - & -0.0064 & 0.0349 & -0.0661 & 0.0382 \\
\hline & 0.0002 & - & -0.0004 & - & - & - & -0.0061 & 0.0357 & -0.0671 & 0.0375 \\
\hline & 0.0002 & - & - & -0.0043 & - & - & -0.0058 & 0.0345 & -0.0673 & 0.0387 \\
\hline & 0.0002 & - & - & - & -0.0142 & - & -0.0051 & 0.0354 & -0.0625 & 0.0461 \\
\hline & 0.0003 & - & - & - & - & 0.0201 & -0.0079 & 0.0365 & -0.0708 & 0.0533 \\
\hline \multirow{5}{*}{ 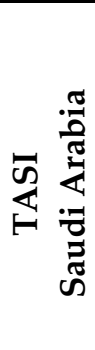 } & 0.0004 & -0.0291 & - & - & - & - & 0.0101 & 0.0719 & -0.0453 & 0.0522 \\
\hline & 0.0004 & - & -0.0313 & - & - & - & 0.0121 & $0.0795^{* * *}$ & -0.0636 & 0.0609 \\
\hline & 0.0005 & - & - & -0.0045 & - & - & 0.0125 & $0.0752^{* * *}$ & -0.0510 & 0.0408 \\
\hline & 0.0005 & - & - & - & -0.0081 & - & 0.0127 & $0.0763^{* * *}$ & -0.0481 & 0.0413 \\
\hline & 0.0005 & - & - & - & - & 0.0010 & 0.0121 & $0.0765^{* * *}$ & -0.0508 & 0.0403 \\
\hline$\varangle 0$ & -0.0002 & 9.33E-05 & - & - & - & - & 0.0038 & $0.0565^{* *}$ & -0.0442 & 0.0518 \\
\hline
\end{tabular}


Journal of Management and Economic Studies, vol.2, issue.3, pp.152-165

\begin{tabular}{|c|c|c|c|c|c|c|c|c|c|}
\hline-0.0002 & - & 0.0007 & - & - & - & 0.0038 & $0.0564^{* *}$ & -0.0439 & 0.0518 \\
\hline-0.0002 & - & - & -0.0019 & - & - & 0.0039 & $0.0560^{* *}$ & -0.0443 & 0.0520 \\
\hline-0.0002 & - & - & - & -0.0042 & - & 0.0041 & $0.0564^{* *}$ & -0.0429 & 0.0525 \\
\hline-0.0002 & - & - & - & - & 0.0086 & 0.0030 & $0.0569^{* *}$ & -0.0458 & 0.0546 \\
\hline
\end{tabular}

$*, * *$ and ${ }^{* * *}$ means significant at $1 \%, 5 \%$ and $10 \%$ level of significance respectively.

Table 5. OLS regression result estimation for BRICS countries during COVID-19 outbreak

\begin{tabular}{|c|c|c|c|c|c|c|c|c|c|c|c|c|}
\hline & C & Bitcoin & Ethereum & Dash & Monero & Ripple & VIX & WTI & GOLD & $\mathrm{CC}$ & CD & $\mathbf{R}^{2}$ \\
\hline \multirow{5}{*}{ 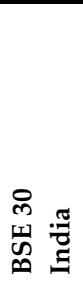 } & 0.0036 & 0.0918 & - & - & - & - & -0.0465 & 0.0207 & 0.180 & -0.0181 & $-0.0411^{* * *}$ & 0.2301 \\
\hline & 0.0034 & - & 0.0721 & - & - & - & -0.0441 & 0.0227 & 0.196 & -0.0187 & $-0.0408^{* * *}$ & 0.2301 \\
\hline & 0.0037 & - & - & 0.0305 & - & - & $-0.0625^{* *}$ & 0.0250 & 0.285 & -0.0191 & $-0.0399^{* * * *}$ & 0.2107 \\
\hline & 0.0031 & - & - & - & 0.0987 & - & $-0.0497^{* * *}$ & 0.0241 & 0.174 & -0.0164 & $-0.0383^{* * *}$ & 0.2622 \\
\hline & 0.0032 & - & - & - & - & 0.1146 & $-0.0467^{* * *}$ & 0.0282 & 0.206 & -0.0181 & -0.0346 & 0.2590 \\
\hline \multirow{5}{*}{ 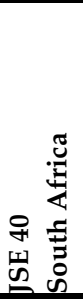 } & 0.0004 & 0.0265 & - & - & - & - & $-0.1036^{*}$ & 0.0182 & $0.769^{*}$ & $-0.0226^{* *}$ & 0.0245 & 0.5531 \\
\hline & 0.0006 & - & 0.0043 & - & - & - & $-0.1084^{*}$ & 0.0191 & 0.798 & $-0.0241^{* *}$ & 0.0250 & 0.5505 \\
\hline & 0.0005 & - & - & 0.0071 & - & - & $-0.1082^{*}$ & 0.0193 & $0.798^{*}$ & $-0.0237^{* *}$ & 0.0255 & 0.5511 \\
\hline & 0.0002 & - & - & - & 0.0384 & - & $-0.1030^{*}$ & 0.0192 & $0.762^{*}$ & $-0.0211^{* *}$ & 0.0247 & 0.5608 \\
\hline & 0.0002 & - & - & - & - & 0.0408 & $-0.1028^{*}$ & 0.0207 & $0.779^{*}$ & $-0.0209^{* *}$ & 0.0256 & 0.5585 \\
\hline \multirow{5}{*}{ 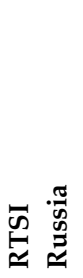 } & -0.0018 & $0.1346^{* *}$ & - & - & - & - & $-0.0640^{* *}$ & $0.0969^{*}$ & $0.382^{* * * *}$ & -0.0114 & 0.0112 & 0.4345 \\
\hline & -0.0017 & - & $0.0906^{* * *}$ & - & - & - & $-0.0659^{* *}$ & $0.0996^{*}$ & $0.430^{* * *}$ & -0.0132 & 0.0103 & 0.4234 \\
\hline & -0.0010 & - & - & 0.0442 & - & - & $-0.0831^{*}$ & $0.1004^{*}$ & $0.529^{* *}$ & -0.0215 & 0.0183 & 0.4110 \\
\hline & -0.0032 & - & - & - & $0.1399^{*}$ & - & $-0.0692^{* *}$ & $0.1037^{*}$ & $0.394^{* * *}$ & -0.0060 & 0.0190 & 0.4693 \\
\hline & -0.0017 & - & - & - & - & $0.1106^{* *}$ & $-0.0750^{* *}$ & $0.1052^{*}$ & $0.489^{* *}$ & -0.0130 & 0.0162 & 0.4283 \\
\hline \multirow{5}{*}{ Wூ } & 0.0039 & 0.0079 & - & - & - & - & -0.0054 & 0.0237 & $0.319^{* *}$ & -0.0141 & 0.0016 & 0.1569 \\
\hline & 0.0003 & - & 0.0050 & - & - & - & -0.0057 & 0.0239 & $0.323^{* *}$ & -0.0141 & 0.0016 & 0.1566 \\
\hline & 0.0003 & - & - & 0.0070 & - & - & -0.0056 & 0.0239 & $0.324^{*}$ & -0.0126 & -5.69 & 0.1579 \\
\hline & 0.0004 & - & - & - & 0.0213 & - & -0.0028 & 0.0237 & $0.304^{* *}$ & -0.0140 & 0.0007 & 0.1652 \\
\hline & 0.0004 & - & - & - & - & 0.0175 & -0.0038 & 0.0243 & $0.318^{* *}$ & -0.0140 & 0.0009 & 0.1605 \\
\hline \multirow[b]{5}{*}{ 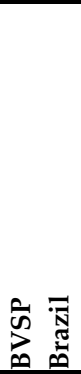 } & 0.0027 & $0.1993^{*}$ & - & - & - & - & $-0.1382^{*}$ & -0.012 & 0.331 & $-0.056^{* *}$ & 0.0104 & 0.5020 \\
\hline & 0.0016 & - & $0.1833^{*}$ & - & - & - & $-0.1232^{*}$ & -0.0083 & 0.314 & $-0.0541^{* *}$ & 0.0118 & 0.5262 \\
\hline & 0.0034 & - & - & 0.0202 & - & - & $-0.1816^{*}$ & -0.0071 & $0.587^{* * *}$ & $-0.0641^{* *}$ & 0.0182 & 0.4391 \\
\hline & 0.0025 & - & - & - & 0.0812 & - & $-0.1714^{*}$ & -0.0050 & $0.503^{* * *}$ & $-0.0567^{* *}$ & 0.0161 & 0.4551 \\
\hline & 0.0023 & - & - & - & - & $0.1349^{* *}$ & $-0.1609^{*}$ & -0.0011 & $0.498^{* * * *}$ & $-.0 .0548^{* *}$ & 0.01753 & 0.4734 \\
\hline
\end{tabular}

Note: CC indicate cumulative number of COVID-19 cases while CD indicate cumulative number of COVID19 deaths. ${ }^{*}{ }^{* *}$ and ${ }^{* * *}$ means significant at $1 \%, 5 \%$ and $10 \%$ level of significance respectively. 
Table 6. OLS regression result estimation for Gulfs countries during COVID-19 outbreak

\begin{tabular}{|c|c|c|c|c|c|c|c|c|c|c|c|c|}
\hline & C & Bitcoin & Ethereum & Dash & Monero & Ripple & VIX & WTI & GOLD & $\mathrm{CC}$ & CD & $\mathbf{R}^{2}$ \\
\hline \multirow{5}{*}{ 完 } & 0.0015 & $0.1113^{* *}$ & - & - & - & - & 0.0441 & $0.0606^{* *}$ & 0.024 & -0.0216 & 0.0043 & 0.2746 \\
\hline & 0.0016 & - & $0.0701^{* * *}$ & - & - & - & $0.0482^{* * *}$ & $0.0630^{* *}$ & 0.072 & -0.0237 & 0.0040 & 0.2559 \\
\hline & 0.0007 & - & - & 0.0822 & - & - & -0.0514 & \begin{tabular}{|l|}
0.0657 \\
\end{tabular} & 0.111 & -0.0191 & 0.0025 & 0.3187 \\
\hline & 0.0015 & & & & $0.1232^{*}$ & & $-0.0479 * *$ & \begin{tabular}{|l}
$0.0641^{*}$ \\
\end{tabular} & 0.027 & -0.0216 & -0.0075 & 0.3346 \\
\hline & 0.0013 & & & & & $0.1414^{*}$ & $\begin{array}{c}- \\
0.0441^{* * *}\end{array}$ & $0.0694^{*}$ & 0.074 & -0.0173 & -0.0074 & 0.3278 \\
\hline \multirow{5}{*}{ 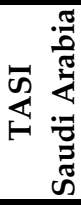 } & -2.19 & -0.0125 & - & - & - & - & $-0.0712^{*}$ & $0.0491^{*}$ & 0.167 & -0.0124 & 0.0128 & 0.3392 \\
\hline & 0.0003 & - & -0.0297 & - & - & - & $-0.0778^{*}$ & $0.0494^{*}$ & 0.198 & -0.0141 & 0.0129 & 0.3478 \\
\hline & \begin{tabular}{|l|}
-0.0004 \\
\end{tabular} & - & - & 0.0120 & - & - & $-0.0654^{*}$ & $0.0488^{*}$ & 0.134 & -0.0106 & 0.0145 & 0.3414 \\
\hline & -0.0005 & - & - & - & 0.0228 & - & $-0.0641^{*}$ & $0.0487^{*}$ & 0.116 & -0.0099 & 0.0146 & 0.3443 \\
\hline & -0.0003 & - & - & - & - & 0.0223 & $-0.0643^{*}$ & $0.0493^{*}$ & 0.129 & -0.0102 & 0.0140 & 0.3423 \\
\hline \multirow{5}{*}{ 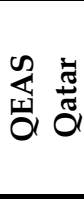 } & -0.0014 & $.08677^{* *}$ & - & - & - & - & -0.0164 & $0.0342^{* *}$ & -0.102 & 0.0037 & 0.0043 & 0.1997 \\
\hline & -0.0008 & - & 0.0393 & - & - & - & -0.0212 & $0.0371^{* *}$ & -0.076 & -0.0022 & 0.0055 & 0.1727 \\
\hline & -0.0014 & - & - & $0.0524^{*}$ & - & - & -0.0234 & $0.0378^{* *}$ & $-0.052^{*}$ & 0.0002 & 0.0113 & 0.2343 \\
\hline & -0.0022 & - & - & - & $0.1096^{*}$ & - & -0.0191 & $0.0367^{* *}$ & -0.089 & 0.0089 & 0.0084 & 0.3074 \\
\hline & -0.0018 & - & - & - & - & $0.1166^{*}$ & -0.0174 & $0.0403^{* *}$ & -0.050 & 0.0070 & 0.0089 & 0.2787 \\
\hline \multirow{5}{*}{$\sum^{\text {⿹ }}$} & -0.0011 & 0.0127 & - & - & - & - & $-0.0288^{*}$ & $0.0203^{* *}$ & 0.113 & -0.0013 & 0.0102 & 0.2917 \\
\hline & -0.0011 & - & 0.0085 & - & - & - & $-0.0290^{*}$ & $0.0205^{* *}$ & $0.118^{* * *}$ & -0.0009 & 0.0093 & 0.2903 \\
\hline & -0.0012 & - & - & 0.0082 & - & - & $-0.0299^{*}$ & \begin{tabular}{|l}
$0.0210^{* *}$ \\
\end{tabular} & $0.122^{* * *}$ & -0.0007 & 0.0106 & 0.2940 \\
\hline & -0.0011 & - & - & - & 0.0128 & - & $-0.0294^{*}$ & \begin{tabular}{|l}
$0.0208^{* *}$ \\
\end{tabular} & 0.115 & -0.0009 & 0.0102 & 0.2959 \\
\hline & -0.0012 & - & - & - & - & 0.0208 & $-0.0278^{*}$ & $0.0214^{* *}$ & $0.114^{* * *}$ & -0.0006 & 0.0108 & 0.3042 \\
\hline \multirow{5}{*}{ 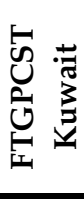 } & -0.0042 & -0.0023 & - & - & - & - & 0.0245 & 0.0110 & 0.339 & -0.0035 & 0.0246 & 0.0661 \\
\hline & -0.0042 & - & -0.0053 & - & - & - & 0.0231 & 0.0111 & $0.344^{* * *}$ & -0.0035 & 0.0246 & 0.0663 \\
\hline & -0.0042 & - & - & -0.0003 & - & - & 0.0250 & 0.0109 & $0.336^{* * *}$ & -0.0036 & 0.0246 & 0.0660 \\
\hline & -0.0041 & - & - & - & -0.0128 & - & 0.02261 & 0.0111 & $0.352^{* * *}$ & -0.0041 & 0.0245 & 0.0675 \\
\hline & -0.0041 & - & - & - & - & -0.0194 & 0.0214 & 0.0105 & $0.350^{* * *}$ & -0.0044 & 0.0243 & 0.0684 \\
\hline \multirow{5}{*}{ 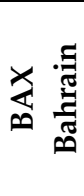 } & -0.0023 & $0.0518^{* *}$ & - & - & - & - & -0.0177 & 0.0174 & -0.021 & 0.0027 & 0.0001 & 0.2170 \\
\hline & \begin{tabular}{|l|}
-0.0024 \\
\end{tabular} & - & $0.0337^{* * *}$ & - & - & - & -0.0190 & $0.0188^{* * *}$ & -0.001 & 0.0028 & 0.0001 & 0.1971 \\
\hline & -0.0025 & - & - & $0.0321^{*}$ & - & - & $-0.0231^{* *}$ & $0.0196^{* * *}$ & 0.023 & 0.0030 & 0.0007 & 0.2360 \\
\hline & -0.0023 & - & - & - & $0.0406^{* *}$ & - & $-0.0227^{* *}$ & $0.0193^{* * *}$ & 0.002 & 0.0028 & 0.0008 & 0.2232 \\
\hline & -0.0023 & - & - & - & - & $0.0455^{* *}$ & $-0.0222^{* *}$ & $0.0207^{* * *}$ & 0.020 & 0.0034 & 0.0001 & 0.2146 \\
\hline
\end{tabular}

Note: CC indicate cumulative number of COVID-19 cases while CD indicate cumulative number of COVID-19 deaths. ${ }^{*}{ }^{* *}$ and ${ }^{* * *}$ means significant at $1 \%, 5 \%$ and $10 \%$ level of significance respectively.

Tables 3 and 4 indicate that for the BRICS countries, cryptocurrencies cannot be regarded as hedges, as all the coefficients are positive and significant; for instance, Bitcoin act as a diversifier for the Indian index and Dash act as a diversifier for the Chinese index. This implies that cryptocurrencies are only effective diversifiers for investors in the developed market. Interestingly, we find evidence of Ripple being a strong hedge for investors in GULF countries like Bahrain and Kuwait. Further, we report a significant negative relationship between VIX, RTSI, and BVSP, indicating that the expected volatility of the US stock market has no effect on the Russian and Brazilian financial markets. In the contrary, we find a significant positive relationship between VIX and FTGPCST, indicating that the expected volatility of the US stock market has an effect on the Kuwait financial market.

Tables 5 and 6 provide the estimated OLS regression results for BRICS and Gulf countries during the covid-19 outbreak. Results of the regression analyses indicate that there is a low significant positive relationship between Bitcoin and Russia, Brazil, Abu-Dhabi, Bahrain, and Qatar during the COVID-19 outbreak. It reveals that cryptocurrencies are rather isolated from other markets. The lower positive coefficient of Bitcoin suggested significant benefits for portfolio diversification 
and risk management when Bitcoin was added. We also find that Russia, Brazil, Bahrain, and Abu-Dhabi have positive relationships with Ethereum indicating the diversification properties of this cryptocurrency. However, Dash act as a diversifier asset for only Qatar and Bahrain investors. Also, Monero and Ripple can be considered as diversifier assets for Russia, and Gulf investors like Abu Dhabi, Bahrain, and Qatar, during the Coronavirus pandemic. This means that cryptocurrencies cannot be considered as safe havens during the pandemic. This result is consistent with Ji et al. (2020) who find that the role of safe haven becomes less effective for most of the cryptocurrencies during the COVID-19 outbreak.

Nevertheless, results indicate that the expected volatility of the US stock market has no effect on the entire BRICS stock index except for china and the entire GCC stock index except for Kuwait financial markets. During the 2020 global pandemic, the results of the regression analyses show that there is a significant positive relationship between GOLD and the majority of the studied indices returns. The higher positive coefficient of GOLD indicates that the yellow metal is neither a hedge nor a safe haven during the COVID-19 pandemic. This result is inconsistent with Baur and Lucy (2010) and Beckmann et al. (2015). This implies that GOLD is only an effective diversifier for investors during the COVID-19 outbreak.

Given that the Coronavirus was initially considered a Chinese crisis, the effects of the outbreak, which previously appeared to be confined to the economic, health, tourism, and industrial sectors of China, spread beyond its native geographic area and extended to the Gulf region. Even though China is the largest oil importer in the Gulf region, the spread of the virus has affected industrial processes, resulting in a drop in oil prices and demand, and thus, the GCC is expected to shake. The oil market in China is arguably one of the most important markets for oil producers. Any drop in oil exports to Chinese refineries will therefore have a significant impact on the revenues of almost all Gulf countries.

After reporting an increase in cases of Coronavirus infection outside China, the world's secondlargest economy, the stock market saw its biggest decline in nearly two years and it is natural to expect negative effects on oil prices and world stock markets, especially as it now affects the world's biggest oil exporter.

Since negotiations between the Organization of Petroleum Exporting countries (OPEC) and its allies failed in the first week of March, oil prices have collapsed. This is reflected in the results, as the WTI dropped to nearly its half price during the COVID-19 pandemic. The results reveal that all of the BRICS indices except India, as well as Saudi Arabia and the United Arab Emirates, are more successful in diversifying their economies than other countries. This is illustrated in Tables 3 and 4, where the regression analyses indicate a low significant positive relationship between WTI price, Saudi Arabia, the United Arab Emirates, and all of the BRICS indices except India before COVID-19. This shows that the impact of lower oil prices reveals the importance of economic diversification.

However, the economies of oil-exporting countries, mainly the Gulf States and Russia, continue to depend on the oil and gas sector for their revenues and exports. In order to reduce the crisis, it must change this pattern of growth through economic diversification policies in order to mitigate and reduce the risk of lower prices. This strategy has proved to be very effective in helping the state overcome market turbulence and protect invested capital from the risks associated with very few assets.

Furthermore, tables 5 and 6 show that there is a significant low positive correlation between the WTI stock price, the GCC stock market, and Russia index during the outbreak of the Coronavirus. 
Finally, our results show that the growth rate of confirmed COVID-19 deaths has no impact on all the GCC stock market returns and all the BRICS stock market returns except for India.

\section{4- Conclusion}

After the outbreak of the coronavirus pandemic, the impact on the global financial and economic system begins to be felt. Using the OLS regression, we focus on the determinants of BRICS and GCC stock market returns during the COVID-19 outbreak. Our results reveal that Bitcoin and Ethereum can generate benefits from portfolio diversification and hedging strategies for Russia, Brazil, Abu-Dhabi, Bahrain, and Qatar financial investors. However, Dash act as a diversifier asset for only Qatar and Bahrain investors. Added to this, Monero and Ripple can be considered as diversifier assets for Russia, and Gulf investors such as Abu Dhabi, Bahrain, and Qatar. This means that none of the cryptocurrencies can act as a safe haven. During this crisis, global stock markets became more volatile, leading investors to panic due to the intense market uncertainty and the plunge in the global stock markets and crude oil prices. Furthermore, the results reveal that Gold is neither hedge nor safe haven but it acts as an effective diversifier for investors during the covid-19 outbreak. Moreover, results indicate that the expected volatility of the US stock market has an effect only on the Kuwait and Chinese financial markets. Finally, our results show that the growth rate of the COVID-19 confirmed cases has impact only on Brazil and South Africa stock market returns.

\section{References}

Aslanidis N., Bariviera A-F., Martínez-Ibañez O., (2019). An analysis of cryptocurrencies conditional cross correlations. Finance Research Letters, 31, 130-137.

Akoum, I., Graham, M., Kivihaho, J., Nikkinen, J., and Omran, M., (2012). Co-movement of oil and stock prices in the GCC region: A wavelet analysis. The Quarterly Review of Economics and Finance, 52, (4), 385-394.

Baur, D.G., Lucey, B.M., (2010). Is gold a hedge or a safe haven? An analysis of stocks, bonds and gold. The Financial. Review. 45 (2), 217-229.

Baur, D.G., \& McDermott, T.K., (2010). Is gold a safe-haven? International evidence. Journal of Banking \& Finance, 34, 1886-1898.

Beckmann, J., Berger, T., Czdaj, R., 2015. Does Gold Act As a Hedge or a Safe Haven for Stocks?A Smooth Transition Approach. Economic Modelling, 48, 16-24.

Basher, S., Sadorsky, P., (2016). Hedging emerging market stock prices with oil, gold, VIX, and bonds: A comparison between DCC, ADCC and GO-GARCH. Energy Economics, 54, (C), 235-247.

Bouri, E., Molnar, P., Azzi, G., Roubaud, D., Hagfors, L., (2017). On the hedge and safe haven properties of Bitcoin: is it really more than a diversifier? Finance Research Letters, 20, 192-198.

Charfeddine L., Benlagha N., Maouchi Y., 2020. Investigating the dynamic relationship between cryptocurrencies and conventional assets: Implications for financial investors. Economic Modelling, 85, 198-217.

Chkili, W., (2016) Dynamic correlations and hedging effectiveness between gold and stock markets: evidence for BRICS countries. Research in International Business and Finance, $38,22-34$.

Dyhrberg, A.H., (2016). Hedging capabilities of Bitcoin. Is it the virtual gold? Finance Research Letters, 16, 139-144. 
Hamed, K., Al-Yahyaee, Mensi, W., Sensoy, A., and Hoon Kang, S., (2019). Energy, precious metals, and GCC stock markets: Is there any risk spillover?, Pacific-Basin. Finance Journal, 56, (C), 45-70.

Ji, Q., Zhang, D., Zhao, Y., (2020). Searching for safe-haven assets during the COVID-19 pandemic. International Review of Financial Analysis. 71, 101526.

Klein, T., Pham Thu, H., Walther, T., (2018). Bitcoin is not the New Gold a comparison of volatility, correlation, and portfolio performance. International Review of Financial Analysis. 59, 105-116.

Maghyereh, A. I., Awartani, B., \& Tziogkidis, P., (2017). Volatility spillovers and cross-hedging between gold, oil and equities: Evidence from the Gulf Cooperation Council countries. Energy Economics, 68, (C), 440-45.

Mensi, M., Hammoudeh, S., Nguyen, D.K., Hoon Kang, S., (2016). Global financial crisis and spillover effects among the U.S. and BRICS stock markets. International Review of Economics \& Finance, 42, (C), 257-276.

Mensi, W., Hkiri, B., Al-Yahyaee, K. H., \& Kang, S. H. (2018). Analyzing time-frequency comovements across gold and oil prices with BRICS stock markets: A VaR based on wavelet approach. International Review of Economics and Finance, 54, 74-102.

Nakamoto, S. (2008). Bitcoin: A Peer-to-Peer Electronic Cash System.

Shahzad, S. J. H., Bouri, E., Roubaud, D., Kristoufek, L., \& Lucey, B. (2019). Is Bitcoin a better safehaven investment than gold and commodities? International Review of Financial Analysis, 63, 322-330.

Urquhart, A., and Zhang, H., (2019). Is Bitcoin a hedge or safe haven for currencies? An intraday analysis. International Review of Financial Analysis, 63, 49-57.

Ulussever, T., and Demirer, R., (2017). Investor herds and oil prices evidence in the Gulf Cooperation Council (GCC) equity markets. Central Bank Review, 17, (3), 77â€“"89

Yonghong, J., Yuyuan, F., Weihua, R., 2019. Risk spillovers and portfolio management between precious metal and BRICS stock markets. Physia A 534, 120993. 